\title{
Long non-coding RNA FER1L4 inhibits cell proliferation and promotes cell apoptosis via the PTEN/AKT/p53 signaling pathway in lung cancer
}

\author{
LANWEI OUYANG $^{1,2^{*}}$, MIN YANG $^{3 *}$, XIAOLIN WANG ${ }^{1}$, JIDAN FAN $^{3}$, \\ XING LIU ${ }^{4}$, YONG ZHANG ${ }^{1,2}$ and YUSHENG SHU ${ }^{1}$ \\ ${ }^{1}$ Department of Thoracic Surgery, Northern Jiangsu People's Hospital, Yangzhou, Jiangsu 225001; \\ ${ }^{2}$ Dalian Medical University, Dalian, Liaoning 116044; ${ }^{3}$ Department of Cardiac Macrovascular Center, \\ Northern Jiangsu People's Hospital, Yangzhou, Jiangsu 225001; ${ }^{4}$ Department of Surgical Oncology, \\ The Second People's Hospital of Yichang, Yichang, Hubei 443000, P.R. China
}

Received December 5, 2019; Accepted March 31, 2020

DOI: $10.3892 /$ or.2020.7861

\begin{abstract}
Long non-coding RNA Fer-1-like protein 4 (FER1L4) has been reported to play crucial regulatory roles in tumor progression and apoptosis. However, its clinical significance and biological role in non-small cell lung cancer (NSCLC) are completely unknown. The purpose of this study was to investigate the expression of lncRNA FER1L4 in plasma and tissues of patients with NSCLC and study the mechanism of proliferation and apoptosis of lung cancer cells. The expression levels of FER1L4 in plasma and tissues of NSCLC patients and cell lines were analyzed via RT-qPCR. The effects of FER1L4 on cell proliferation, migration and invasion were analyzed by CCK- 8 , wound healing and Transwell assays, respectively. The expression levels of related proteins were detected by western blot assay, while cell apoptosis was determined by Hoechst staining and flow cytometry. The results revealed that FER1L4 was significantly downregulated in NSCLC plasma and tissues and lung cancer cell lines compared to corresponding controls. Moreover, a significant decrease of cell proliferation, migration and invasion were observed in FER1L4-overexpressed cells. FER1L4 could promote phosphatase and tension homolog deleted on chromosome ten (PTEN) and p53 expression, inhibit AKT phosphorylation expression, thus increasing the proportion of apoptotic cells. The present study indicated that FER1L4 may inhibit cell proliferation and promote apoptosis of NSCLC cells via the PTEN/AKT/p53 pathway, which provides a better
\end{abstract}

Correspondence to: Dr Yusheng Shu, Department of Thoracic Surgery, Northern Jiangsu People's Hospital, 98 West Nantong Road, Yangzhou, Jiangsu 225001, P.R. China

E-mail: shuyusheng2019@163.com

*Contributed equally

Key words: IncRNA FER1L4, NSCLC, PTEN/AKT/p53, cell proliferation, cell apoptosis understanding of the pathogenesis of NSCLC and may provide a novel potential therapeutic target for clinical treatment.

\section{Introduction}

Lung cancer remains the most common malignancy with the highest morbidity and mortality in the world (1). According to a 2015 research study of China, the incidence rate of lung cancer ranks first among men and second among women (2). Lung cancer can be divided into two main types: Small-cell lung cancer (SCLC) and non-small cell lung cancer (NSCLC) according to the pathological classification of lung cancer in clinical practice. NSCLC accounts for $\sim 85 \%$ of all lung cancer patients, with a five-year survival as low as $15 \%$ (3). Currently, with the continuous development of medicine, great progress has been made in the diagnosis and treatment of lung cancer, however, some methods exhibit toxicity-related side effects and present limitations in their application, which seriously threaten the safety of human life (4). Therefore, it is urgent to identify new diagnosis and treatment methods for lung cancer.

Long non-coding RNAs (lncRNAs) are a class of RNAs that do not encode proteins and have transcripts longer than 200 nucleotides. Current studies have confirmed that lncRNAs are closely related to the occurrence and the development of various human malignant tumors (5). In the process of tumor formation, IncRNAs play an important role in the function of oncogenes and tumor suppressor genes (6). Some lncRNAs are differentially expressed in tumor and normal tissues, which may be involved in the occurrence and development of tumors (7-10). Fer-1-like protein 4 (FER1L4), as an important member of lncRNAs, is closely related to the occurrence and development of tumors. Studies have reported that lncRNA FER1L4 knockout in liver cancer cells promoted cell proliferation and invasion $(11,12)$. Yue et al $(13)$, revealed that lncRNA FER1L4 inhibited the formation of colon cancer and predicted the prognosis of patients. Liu et al (14), confirmed that the expression level of FER1L4 could be an important indicator for early diagnosis of gastric cancer. However, the role of IncRNA FER1L4 in NSCLC remains unclear. 
From literature review, it was revealed that lncRNA FER1L4 inhibited the process of osteosarcoma cells by targeting microRNA-18a-5p (miR-18a-5p) to release the gene phosphatase and tension homolog deleted on chromosome ten (PTEN) (15). In addition, FER1L4 was revealed to inhibit proliferation, invasion and migration of hepatocellular carcinoma (11). Futhermore, research has revealed that upregulating IncRNA FER1L4 suppressed the proliferation and migration of the hepatocellular carcinoma via regulation of the PI3K/AKT signaling pathway (12). However, the expression of FER1L4 in the plasma and tissues of patients with NSCLC and the role of PTEN/AKT/p53 signaling proteins in the proliferation, invasion and migration of NSCLC are still unknown. In the present study, the expression of IncRNA FER1L4 was examined in the plasma and tissues of patients with NSCLC, and it was investigated whether lncRNA FER1L4 inhibited the proliferation of lung cancer cells and promoted apoptosis through the PTEN/AKT/p53 signaling pathway.

\section{Materials and methods}

Tumor tissues and blood samples. A total of 30 tumor tissues and blood samples were obtained from NSCLC patients (15 females and 15 males; age range, 18-45 years) who received surgical treatment from January to April in 2019 at Northern Jiangsu People's Hospital. Blood and tissues of 31 healthy volunteers were used as the control group. None of the patients had received neoadjuvant therapy or endocrine therapy before the surgery. The present study was approved by the Ethics Committee of Northern Jiangsu People's Hospital, and all patients provided written informed consent. All of the procedures were in compliance with The Declaration of Helsinki and relevant policies in China.

Cell culture. All cell lines used in this study, including BEAS-2B (cat. no. SCSP-5067), H1975 (cat. no. SCSP-597), H23 (cat. no. SCSP-581), A549 (cat. no. TCHu150) and H1299 (cat. no. SCSP-589), were all obtained from the Type Culture Collection of the Chinese Academy of Sciences, and were cultured in DMEM supplemented with $10 \%$ FBS (both from Gibco; Thermo Fisher Scientific, Inc.) at $37^{\circ} \mathrm{C}$ with $5 \% \mathrm{CO}_{2}$. The HBE cell line is a human bronchial epithelial cell line, and H1975, H23, A549 and H1299 are all lung cancer cell lines.

RNA extraction and reverse transcription-quantitative polymerase chain reaction $(R T-q P C R)$. Total RNA from tumor tissues, blood samples and cultured cells were extracted using TRIzol ${ }^{\circledR}$ reagent (Invitrogen; Thermo Fisher Scientific, Inc.), following the manufacturer's protocol. The RNA concentration and quantification were assessed using a Nanodrop spectrophotometer (Thermo Fisher Scientific, Inc.). Reverse transcription of RNA into complementary DNA (cDNA) was performed using a PrimeScript ${ }^{\mathrm{TM}}$ reverse transcription reagent kit (Takara Biotechnology Co., Ltd.), according to the manufacturer's protocol. Subsequently, cDNA was analyzed using a TaqMan ${ }^{\circledR}$ Universal PCR Master Mix kit (Thermo Fisher Scientific, Inc.), according to the manufacturer's protocol. Amplification conditions were as follows: $95^{\circ} \mathrm{C}$ for $10 \mathrm{~min}$, followed by 40 cycles at $95^{\circ} \mathrm{C}$ for $10 \mathrm{sec}$ and $60^{\circ} \mathrm{C}$ for $60 \mathrm{sec}$. Primer sequences used in PCR were obtained from GenScript.
The primer sequences were as follows: FER1L4 forward, 5'-AATGTGGGCTTCCAGGAAC-3' and reverse, 5'-CAC CAGAAAGTTCCACGTC-3'; GAPDH forward, 5'-GGTCGG AGTCAACGGATTTG-3' and reverse, 5'-GGAAGATGGTGA TGGGATTTC-3'. The relative gene expression level quantification was analyzed using the $2^{-\Delta \Delta \mathrm{Cq}}$ method, and normalized to GAPDH expression (16).

Cell transfection. One day prior to transfection, A549 cells $\left(1 \times 10^{5}\right.$ cells/well) were seeded into 6 -well plates and cultured at $37^{\circ} \mathrm{C}$ in an atmosphere containing $5 \% \mathrm{CO}_{2}$. Subsequently, the cells were transfected with FER1L4 or negative control (NC) using Lipofectamine ${ }^{\circledR} 2000$ (Invitrogen; Thermo Fisher Scientific, Inc.), according to the manufacturer's protocol. Cells of the blank control group (Control) did not receive any treatment. When cells were incubated for $48 \mathrm{~h}$, they were used for the following experiments. Transfection efficiency was detected by reverse transcription-quantitative polymerase chain reaction (RT-qPCR).

Cell Counting Kit-8 (CCK-8) assay. The proliferation of cells was assessed using CCK-8 kit (Dojindo Molecular Technologies, Inc.) according to the manufacturer's instructions. In brief, cells were seeded into 96 -well plates, and trypsinized to prepare a cell suspension with a density of $2 \times 10^{3}$ cells $/ \mathrm{ml}$. After $4 \mathrm{~h}$ of incubation, $10 \mu \mathrm{l}$ of CCK-8 solution was added to each well and further incubated for $2 \mathrm{~h}$ at $37^{\circ} \mathrm{C}$. The absorbance at $450 \mathrm{~nm}$ was measured using a microplate reader (BioTek Instruments, Inc.).

Wound healing assay. Cell migration was determined by wound-healing assays. Briefly, transfected cells were plated in 12-well plates at a density of $1 \times 10^{5}$ cells/well. Once cells reached $80 \%$ confluence, the medium was replaced by serum-free DMEM and cells were incubated at $37^{\circ} \mathrm{C}$ overnight before initiating the experiment. A wound was created on the surface of the cell monolayer using a $200-\mu 1$ pipette tip. The cells were then rinsed twice with serum-free medium in order to remove free-floating cells and debris. An inverted microscope (magnification x20; BX51; Olympus Corporation) was used to monitor cells along the edge of the scratch. Cell mobility $=(0-\mathrm{h}$ scratch width -24-h scratch width)/0-h scratch width.

Cell invasion assay. To assess the invasion of transfected cells, 24-well Transwell plates (Corning, Inc.) with $8-\mu \mathrm{m}$ pore inserts were coated with Matrigel (BD Biosciences). A total of $200 \mu \mathrm{l}$ serum-free medium cell suspension containing $5 \times 10^{4}$ cells $/ \mathrm{ml}$ was added to the upper chamber and $600 \mu 1$ DMEM containing $10 \%$ FBS was added to the lower compartment. After $24 \mathrm{~h}$ of incubation at $37^{\circ} \mathrm{C}$ with $5 \% \mathrm{CO}_{2}$, the Matrigel and the cells remaining on the upper chamber were removed by a cotton-tipped swab. The filters were fixed in $4 \%$ formaldehyde for $10 \mathrm{~min}$ at room temperature and stained with $0.1 \%$ crystal violet solution for $30 \mathrm{~min}$ at room temperature. The cells in five random fields (magnification x20) were observed under an inverted microscope (Olympus Corporation).

Western blotting. The protein levels of Ki67, proliferating cell nuclear antigen (PCNA), matrix metalloproteinase-2 (MMP2), MMP9, phosphatase and tension homolog deleted 
A

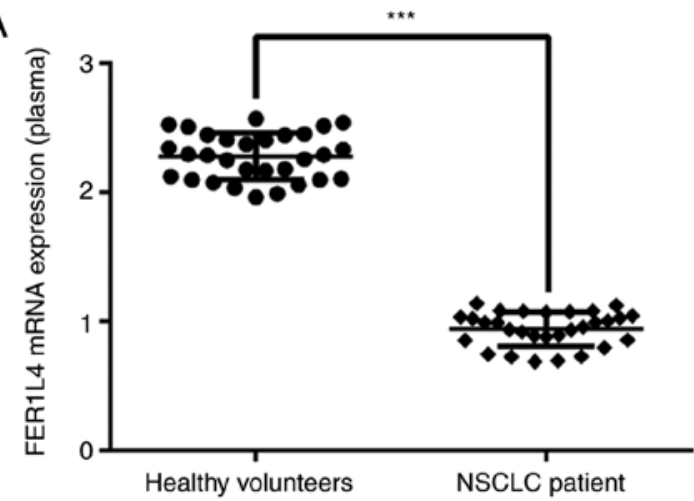

B

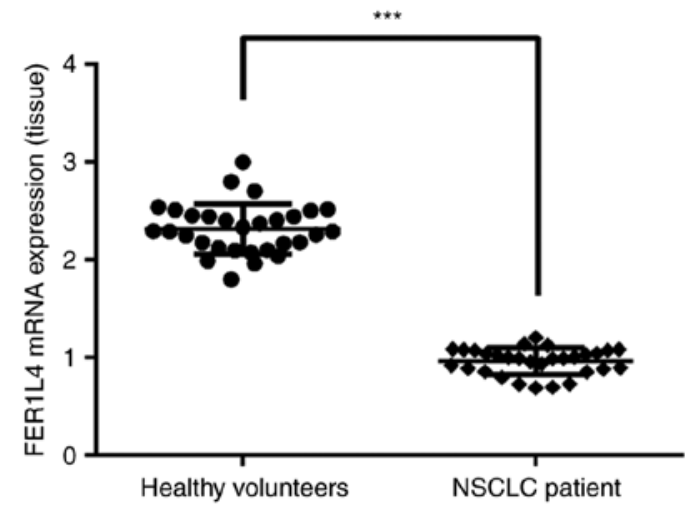

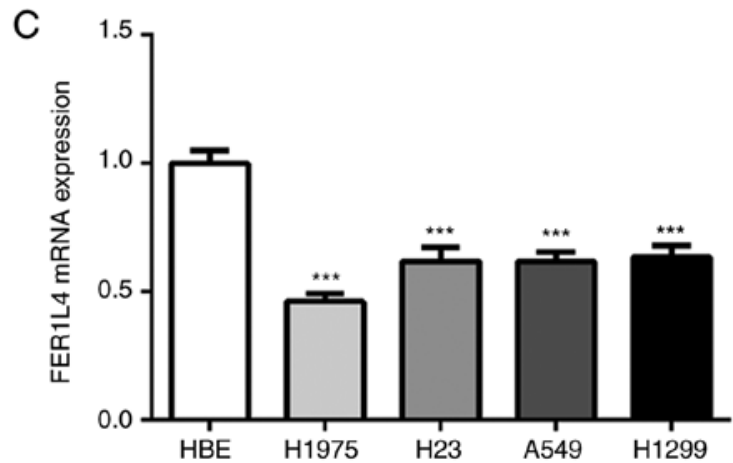

Figure 1. IncRNA FER1L4 expression is downregulated in the plasma of patients with lung cancer and in lung cancer cell lines. (A and B) The expression of FER1L4 in plasma and lung tissue was detected by RT-qPCR. ${ }^{* * *} \mathrm{P}<0.001$ vs. healthy volunteers. (C) The expression of FER1L4 in normal cells (HBE) and lung cancer cell lines (H1975, H23, A549 and H1299) was detected by RT-qPCR. ${ }^{* * *}$ P<0.001 vs. HBE. FER1L4, Fer-1-like protein 4.

on chromosome ten (PTEN), p-AKT, AKT, Bcl-2, BAX, p53, cleaved caspase- 3 and cleaved caspase- 9 were assessed by western blotting. In brief, the total cell proteins were extracted from cells using SDS lysis buffer (Cell Signaling Technology, Inc.). Subsequently, a BCA assay (Thermo Fisher Scientific,Inc.) was used to detect the protein concentrations. Subsequently, equal amounts of protein samples ( $25 \mu \mathrm{g} / \mathrm{lane})$ were separated by $10 \%$ SDS-PAGE and then transferred onto polyvinylidene difluoride membranes. Following blocking with 5\% non-fat milk at room temperature for $2 \mathrm{~h}$, the membranes were incubated with the primary antibodies against Ki67 (1:1,000; product no. 9449T), PCNA $(1: 1,000$; product no. 13110T), MMP2 $(1: 1,000$; product no. 40994T), MMP9 (1:1,000; product no. 13667T), PTEN (1:1,000; product no. 9188T), p-AKT $(1: 1,000$; product no. 13038T), AKT (1:1,000; product no. 4685T), Bcl-2 (1:1,000; product no. 15071T), BAX (1:1,000; product no. 5023T), p53 (1:1,000; product no. 2527T), cleaved caspase-3 (1:1,000; product no. 9664T) and cleaved caspase-9 (1:1,000; product no. 9509T) obtained from Cell Signaling Technology, Inc. at $4^{\circ} \mathrm{C}$ overnight. Then, the membranes were incubated with secondary antibodies: Anti-mouse IgG, HRP-linked antibody (1:5,000; product no. 7076S; Cell Signaling Technology, Inc.) at room temperature for $2 \mathrm{~h}$. GAPDH (1:5,000; product no. 5174T; Cell Signaling Technology, Inc.) was used as the internal control. At the end of the experiment, the protein bands were visualized with an enhanced chemiluminescence detection system (Super Signal West Dura Extended Duration Substrate; Pierce; Thermo Fisher Scientific, Inc.) and ImageJ software (v1.46; National Institutes of Health) was used to analyze the fold-changes of the protein levels.
Cell apoptosis assay. Transfected cells were plated in 6-well plates at a density of $1 \times 10^{5}$ cells/well. Seventy-two hours after adding $2 \mu \mathrm{m}$ of PTEN inhibitor SF1670 to transfected cells, cells were collected for Annexin V/PI double staining (Annexin V-FITC; Vazyme Biotech Co., Ltd.). The staining procedure was conducted according to the manufacturer's instructions. The activity of Annexin V/PI was then detected by flow cytometry (FACSCalibur; BD Biosciences) and quantified by FlowJo software (v7.6.1; FlowJo LLC).

Hoechst staining. Transfected cells were plated in 6-well plates at a density of $1 \times 10^{5}$ cells/well. Seventy-two hours after adding $2 \mu \mathrm{m}$ of PTEN inhibitor SF1670 to transfected cells, cells were incubated with $1 \mu \mathrm{g} / \mu 1$ Hoechst 33342 (Beyotime Institute of Biotechnology) for $15 \mathrm{~min}$, washed twice with $\mathrm{dd}_{2} \mathrm{O}$, and observed under a fluorescence microscope (magnification, x20).

Statistical analysis. Data are expressed as the mean \pm standard deviation. SPSS v17.0 statistical software (SPSS, Inc.) was used for all statistical analyses. Comparisons between groups were analyzed by Student's t-test or one-way analysis of variance (ANOVA) followed by Tukey's post hoc test. $\mathrm{P}<0.05$ was considered to indicate a statistically significant difference.

\section{Results}

IncRNA FERIL4 expression is downregulated in plasma of patients with lung cancer and lung cancer cell lines. The blood and tissues of lung cancer patients and normal volunteers 
A

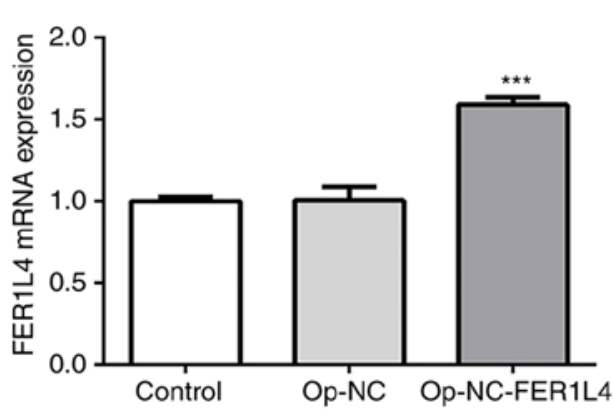

C

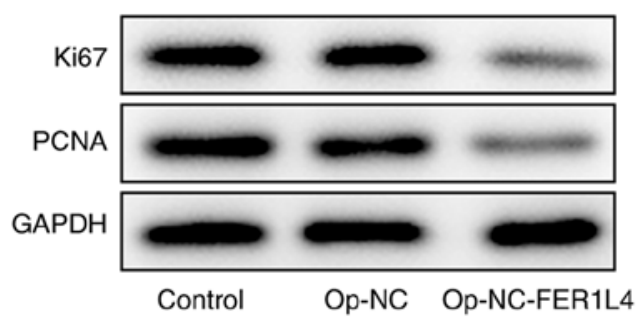

B
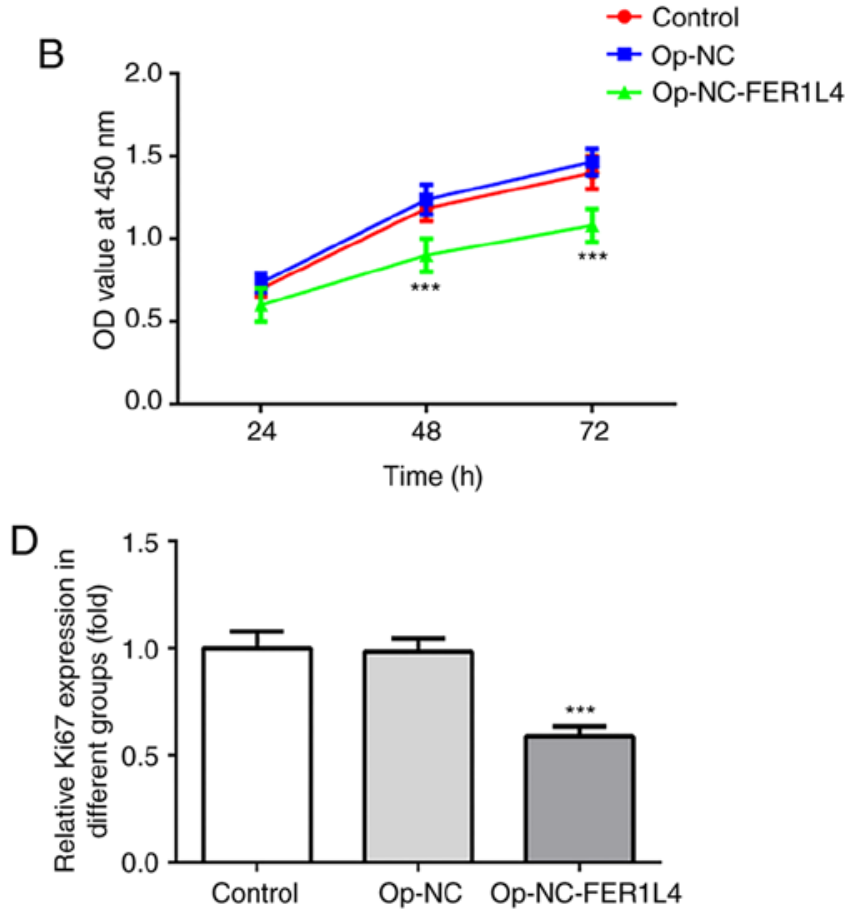

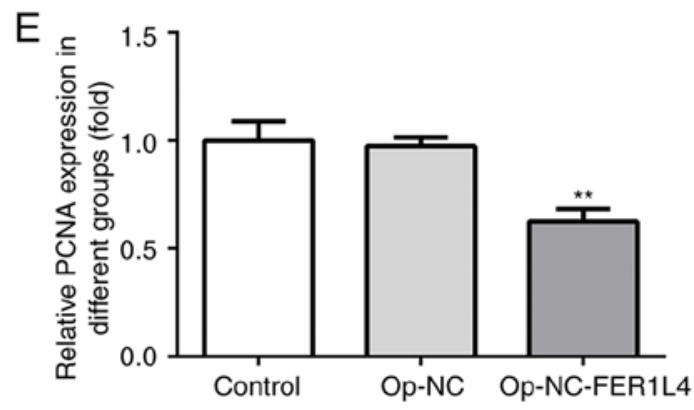

Figure 2. Overexpression of 1ncRNA FER1L4 inhibits the proliferation of A549 cells. Overexpressed plasmids of FER1L4 were constructed by plasmid transfection, and Op-NC was used as a negative control for Op-NC-FER1L4. (A) Overexpression of FER1L4 was detected by RT-qPCR. (B) A CCK-8 was used to detect cell proliferation. (C) The expression levels of proliferation protein Ki67 and PCNA were detected by western blotting. (D) Statistical analysis of the Ki67 protein. (E) Statistical analysis of the PCNA protein. ${ }^{* *} \mathrm{P}<0.01$ and ${ }^{* * * *} \mathrm{P}<0.001$ vs. Op-NC. FER1L4, Fer-1-like protein 4; CCK-8, Cell Counting Kit-8; PCNA, proliferating cell nuclear antigen.

were collected, and the expression of FER1L4 in plasma and tissues was detected by RT-qPCR. A significant decrease of the expression level of FER1L4 was observed in plasma and tissues of lung cancer patients (Fig. 1A and B). Then, in order to conduct the functional studies on FER1L4, its expression in the HBE cell line which was considered as a control and four lung cancer cell lines (H1975, H23, A549 and H1299) was also detected. Similarly, FER1L4 expression was significantly decreased in H1975, H23, A549 and H1299 cells compared to HBE cells (Fig. 1C). After comprehensive consideration, since A549 cells were significantly decreased and are easily cultured this cell line was selected for the following experiments.

OverexpressionoflncRNAFERIL4 inhibitsproliferationof A549 cells. The transfection efficiency of the cells was detected, and as revealed in Fig 2A, compared to the overexpression-NC group, the expression of FER1L4 in the overexpression-NC-FER1L4 group was significantly increased. The proliferation rate of cells was detected by CCK-8 assay (Fig. 2B) and the expression levels of proliferation-related proteins Ki67 and PCNA were detected by western blotting (Fig. 2C). The data revealed that compared to the overexpression-NC group, the proliferation rate and the expression levels of Ki67 and PCNA proteins were significantly decreased in lung cells, in which lncRNA FER1L4 was overexpressed (Fig. 2B-E). The results indicated that overexpression of IncRNA FER1L4 inhibited the proliferation of A549 cells.

Overexpression of IncRNA FERIL4 inhibits the invasion and migration abilities of A549 cells. To detect the effect of IncRNA FER1L4 on the invasion and migration of A549 cells, wound healing (Fig. 3A and B) and Transwell (Fig. 3C and D) assays were employed. Western blotting was used to detect the expression of invasion- and migration-related proteins MMP2 and MMP9 (Fig. 3E). The results revealed that overexpression of lncRNA FER1L4 significantly reduced the invasion and migration abilities of cells, as well as the expression levels of MMP2 and MMP9.

Overexpression of IncRNA FERIL4 activates PTEN and inhibits the phosphorylation of AKT. The expression of 

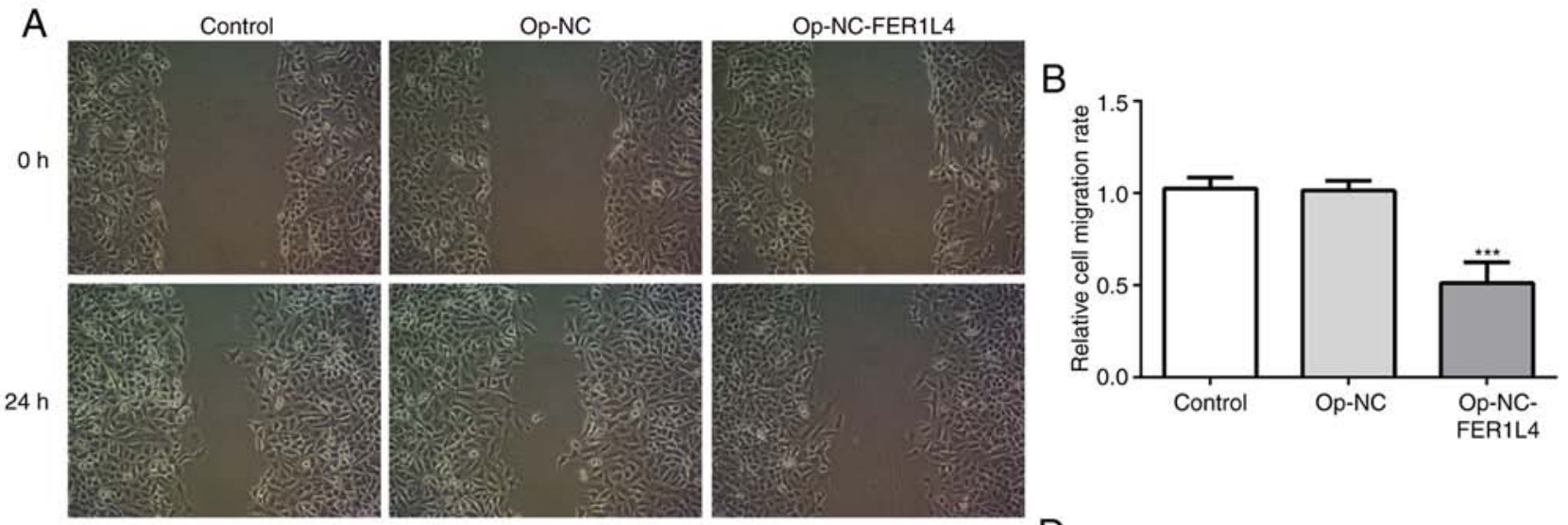

C
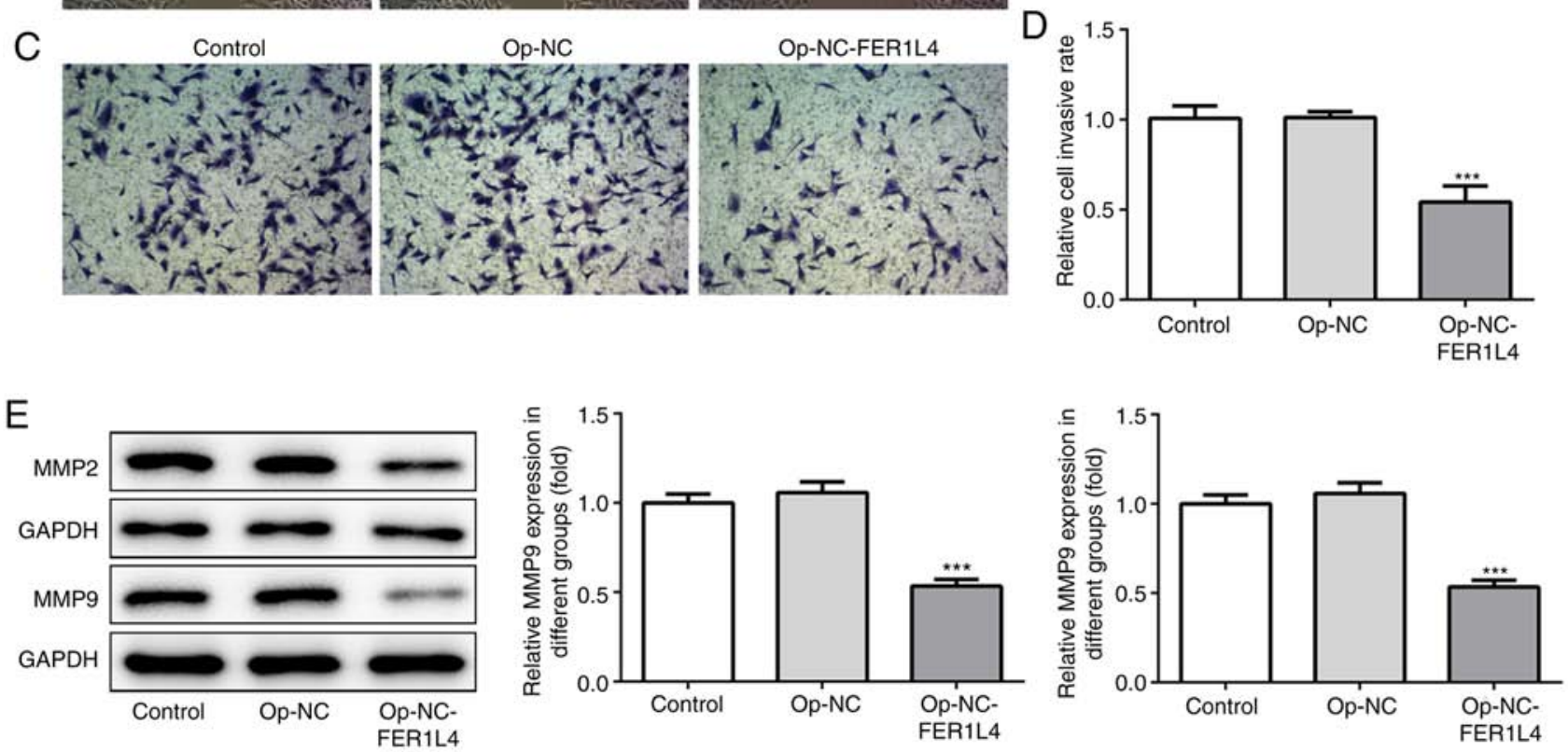

Figure 3. Overexpression of 1ncRNA FER1L4 inhibits the invasion and migration abilities of A549 cells. (A) Migratory activity of A549 cells was evaluated with a scratch assay at different time-points (0 and $24 \mathrm{~h}$ ). Magnification x20. (B) The relative rate of cell migration. (C) Invasion activity of A549 cells was evaluated by Transwell assay (magnification x20). (D) The relative rate of cell invasion. (E) The expression levels of MMP2 and MMP9 were detected by western blotting (left image). Densitometric analysis of the western blot results (middle and right images). ${ }^{* * *} \mathrm{P}<0.001$ vs. Op-NC. FER1L4, Fer-1-like protein 4; MMP2, matrix metalloproteinase-2; MMP9, matrix metalloproteinase-9.
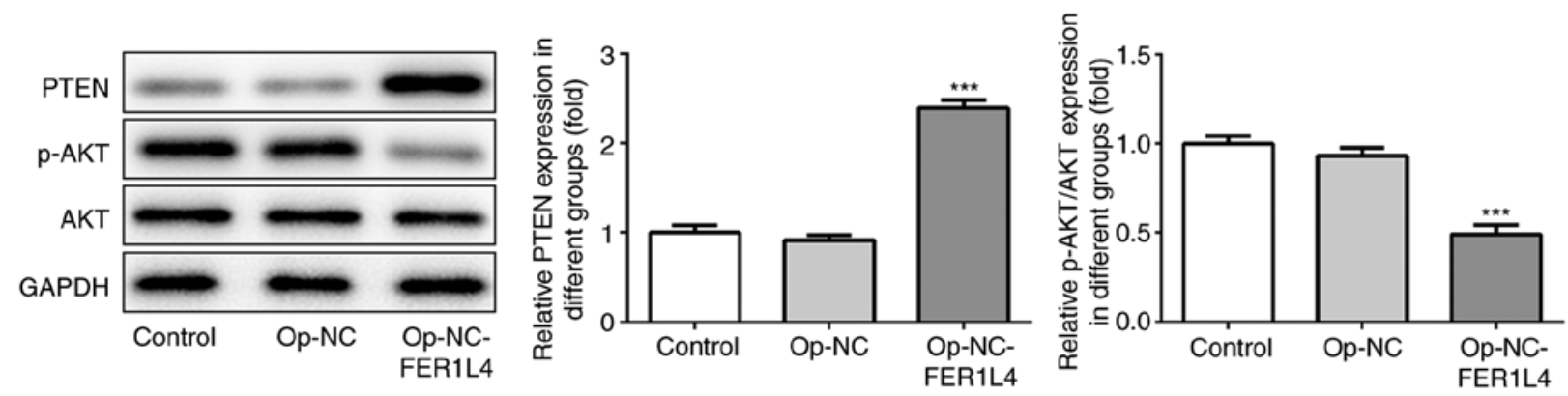

Figure 4. Overexpression of lncRNA FER1L4 activates PTEN and inhibits the phosphorylation of AKT. The expression levels of pathway-related proteins PTEN, p-AKT and AKT were detected by western blotting (left image). Densitometric analysis of the western blot results (middle and right images). ${ }^{* * *} \mathrm{P}<0.001$ vs. Op-NC. FER1L4, Fer-1-like protein 4; PTEN, phosphatase and tension homolog deleted on chromosome ten.

PTEN protein was detected by western blotting, and it was revealed that in cells that overexpressed 1ncRNA FER1L4, PTEN was significantly increased compared with the overexpression-NC group. Additionally, the protein levels of total and phosphorylated AKT (p-AKT) were further analyzed to explore FER1L4 regulation on AKT signaling.
The results revealed that FER1L4 could also inhibit AKT phosphorylation, since a decreased level of p-AKT was observed in FER1L4-overexpressed cells (Fig. 4). These results revealed that FER1L4 regulated the expression of PTEN protein and thus inhibited the expression of AKT phosphorylation. 
Overexpression of LncRNA FERIL4 promotes apoptosis of lung cancer cells by activating PTEN. AKT signaling pathway is closely related to apoptosis $(17,18)$. We previously revealed that overexpressed FER1L4 regulated the PTEN protein and inhibited the phosphorylation of the AKT protein, which may also be related to apoptosis. Therefore, PTEN inhibitor SF1670 was added into the cells, and apoptosis was detected by Hoechst staining (Fig. 5A) and flow cytometry (Fig. 5B and C). Compared with the Control, there was no difference in the apoptosis of Op-NC. Compared with the Op-NC group, the apoptosis rate in Op-NC-FER1L4 was significantly increased after overexpression of FER1L4, but compared with the Op-NC-FER1L4 group, the cell apoptosis was decreased after further SF1670 administration. In addition, the expression levels of apoptosis-related proteins were detected by western blotting (Fig. 6). The results revealed that compared with the OP-NC group, FER1L4 increased cell death with a reduction in apoptosis-related protein $\mathrm{Bcl}-2$ and an increase in BAX, p53, cleaved caspase- 3 and cleaved caspase-9. However, compared with the Op-NC-FER1L4 group, after the addition of SF1670, cell death was decreased, with an increase in apoptosis-related protein Bcl-2, and a decrease in BAX, p53, cleaved caspase-3 and cleaved caspase-9 (Fig. 6). These results indicated that lncRNA FER1L4 promoted lung cancer cell apoptosis via the PTEN/AKT/p53 signaling pathway.

\section{Discussion}

Lung cancer is currently the most common cancer type in the world, accounting for $11.6 \%$ of all cancer cases. It is one of the most malignant tumors with the fastest increase in morbidity and mortality and the greatest threat to human health and life (3). The World Health Organization classifies lung cancer into two major groups, namely non-small cell lung cancer and small-cell lung cancer. NSCLC is more common, including squamous cell carcinoma, adenocarcinoma and large cell lung cancer as well as several others (19). Various chemotherapy and targeted therapies for lung cancer have been studied in the past decade, but most NSCLC cases metastasize and relapse.

lncRNAs are important regulators of gene expression at both transcriptional and post-transcriptional levels. In addition, lncRNAs have been revealed to be involved in regulating the formation, proliferation, invasion and metastasis of tumors, which is closely related to the occurrence and development of tumors $(20,21)$.

Current studies have revealed that the occurrence and development of NSCLC are related to the aberrant expression of lncRNAs. There were 953 aberrant lncRNAs expressed in lung adenocarcinoma and 1,014 aberrant lncR NAs expressed in lung squamous cell carcinoma (22). Pan et al (23), revealed that the expression of FAL1 was significantly upregulated in $78 \%$ of NSCLC cells. Lu et al (24), confirmed that the upregulated expression of SNHG1 promoted the proliferation and migration of NSCLC cells through the further action of miR-145-5p on the expression of MDTH.

lncRNA FER1L4 is an important member of the lncRNA family, and literature has demonstrated that FER1L4 can serve as a cancer suppressor gene to inhibit the occurrence and development of cancer (6). In addition, it can also inhibit the occurrence of gastrointestinal cancer (14). The expression of FER1L4 in colon cancer was revealed to be closely related to the depth of tumor invasion, vascular invasion and lymph node metastasis, and played an important role in suppressing oncogenesis (13). However, there are few studies on the role of lncRNA FER1L4 in NSCLC, thus, the present study innovatively examined the expression of FER1L4 in tissues and plasma of patients with NSCLC and it was revealed that the expression of FER1L4 was significantly reduced. In addition, it was revealed that the expression of FER1L4 in lung cancer cell lines H1975, H23, A549 and H1299 was also significantly decreased.

Targeted researches on tumors are usually conducted by examining the effects of drugs or targeted genes on the proliferation, invasion and migration of tumor cells. lncRNA FER1L4 can inhibit the proliferation, invasion and migration of various tumor cells, such as liver cancer cells, glioma cells, gastric cancer cells and endometrial cancer cells $(12,25,26)$. However, the effect of FER1L4 on NSCLC cells is unknown. Therefore, A549 cells were selected in the present study to further detect the effects of FER1L4 on cell proliferation, invasion and migration. The results revealed that overexpression of lncRNA FER1L4 resulted in decreased proliferation, invasion and migration. This suggests that FER1L4 inhibits the proliferation, invasion and migration of NSCLC cells.

Qiao et al demonstrated that promotion of the expression of PTEN and inhibition of the phosphorylation of AKT signaling pathways inhibited proliferation and migration of endometrial cells (26). FER1L4 was also revealed to regulate the PI3K/AKT signaling pathway to suppress the occurrence and development of liver cancer $(8,27,28)$. In addition, lncRNA FER1L4 inhibited the progression of osteosarcoma cells by targeting miR-18a-5p to release the expression of PTEN (15). Our previous experiments confirmed that lncRNA FER1L4 could inhibit cell proliferation, invasion and migration, but whether it functions through the PTEN/AKT signaling pathway remains to be further studied. Therefore, the expression levels of proteins PTEN, p-AKT and AKT were detected in NSCLC cells and it was revealed that the overexpression of lncRNA FER1L4 significantly increased the expression of PTEN protein and decreased the expression of $\mathrm{p}$-AKT. It was thus concluded that overexpression of lncRNA FER1L4 activated PTEN and inhibited the phosphorylation of AKT, thereby inhibiting cell proliferation, invasion and migration.

Notably, the AKT signaling pathway plays an important role in cell proliferation and apoptosis (29). Furthermore, PTEN was revealed to regulate and promote apoptosis by mediating the AKT pathway (30). Therefore, the effect of FER1L4 overexpression on cell apoptosis was also investigated in the present study. The results revealed that overexpression of lncRNA FER1L4 significantly decreased the cell apoptosis rate and Bcl-2 protein expression, while BAX, cleaved caspase-3, cleaved caspase- 9 protein expression was increased. After the addition of PTEN inhibitor SF1670, the opposite results were obtained. It is well known that p53 is a tumor suppressor gene with the highest tumor suppressor rate in humans, and the apoptotic process is regulated by p53 and other apoptosis-promoting factors (31). Based on this fact, the expression of p53 protein was also assessed, and the results were consistent with the results of pro-apoptotic proteins, BAX, cleaved caspase-3 and cleaved caspase-9. In addition, in a study by Gao et al (32) the role of FER1L4 in lung cancer was examined. Their study revealed 
A
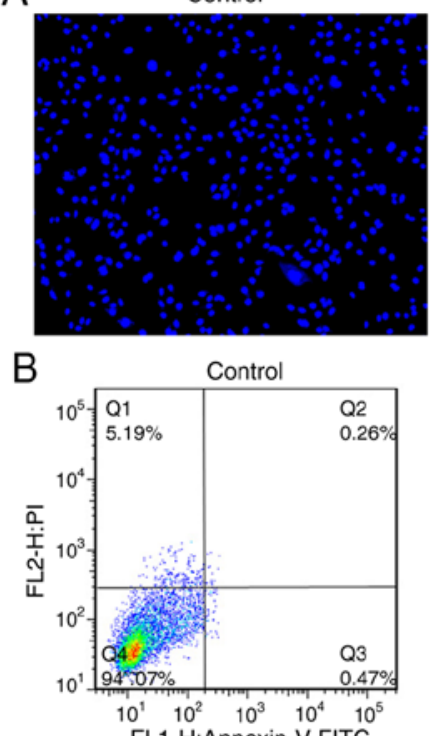

FL1-H:Annexin-V FITC
Op-NC
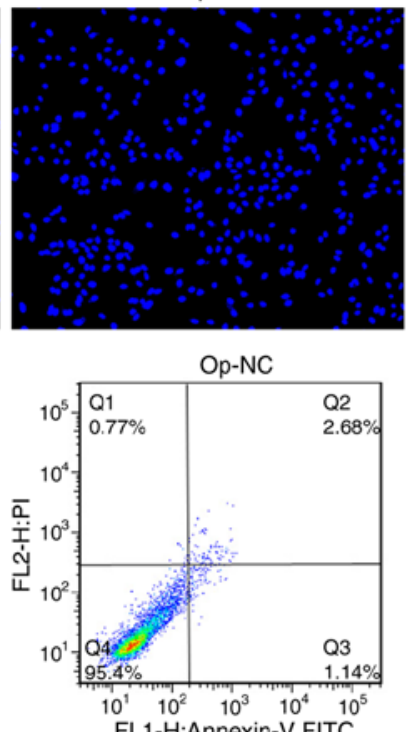

C
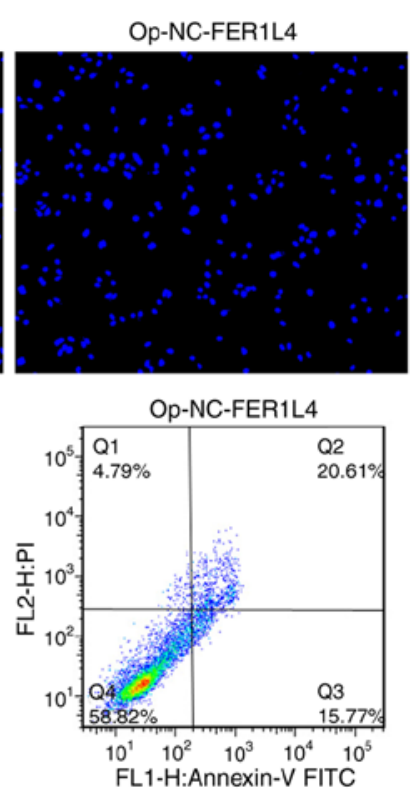

FL1-H:Annexin-V FITC
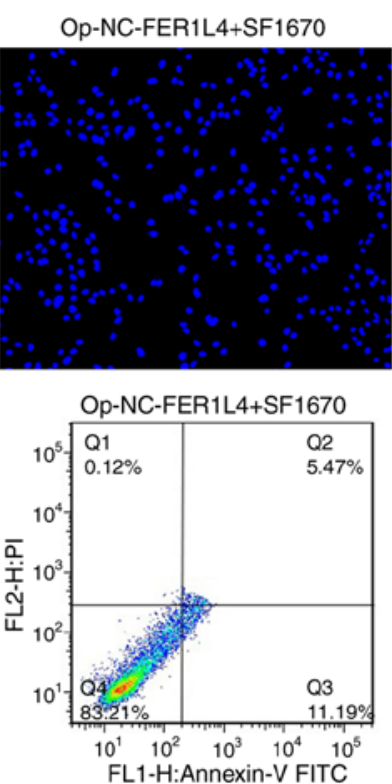

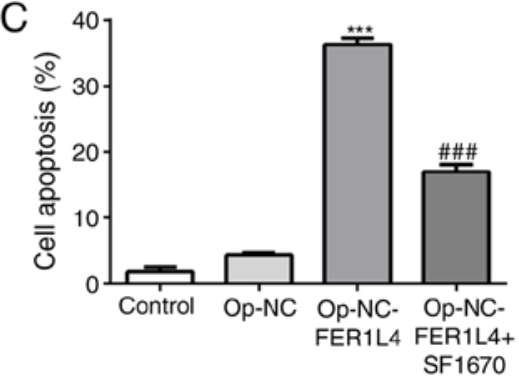

Figure 5. Overexpression of 1ncRNA FER1L4 promotes apoptosis of lung cancer cells by activating PTEN. Overexpressed plasmids of FER1L4 were constructed by plasmid transfection and then the PTEN inhibitor SF1670 was added. Cell apoptosis was detected by (A) Hoechst staining and (B and C) flow cytometry.

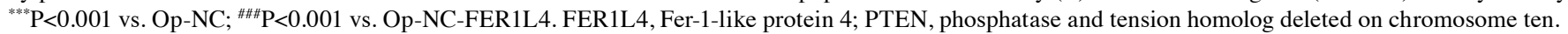
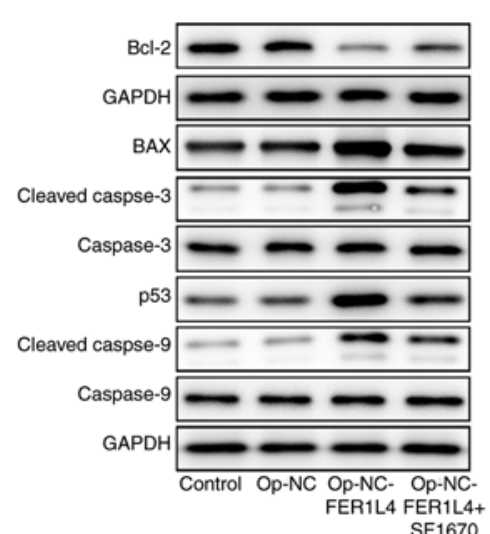

SF1670

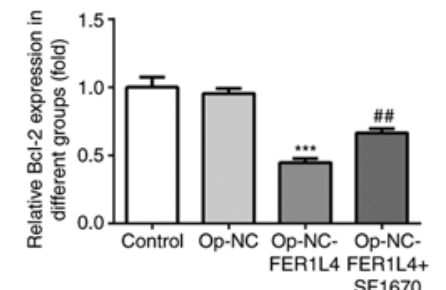

SF1670
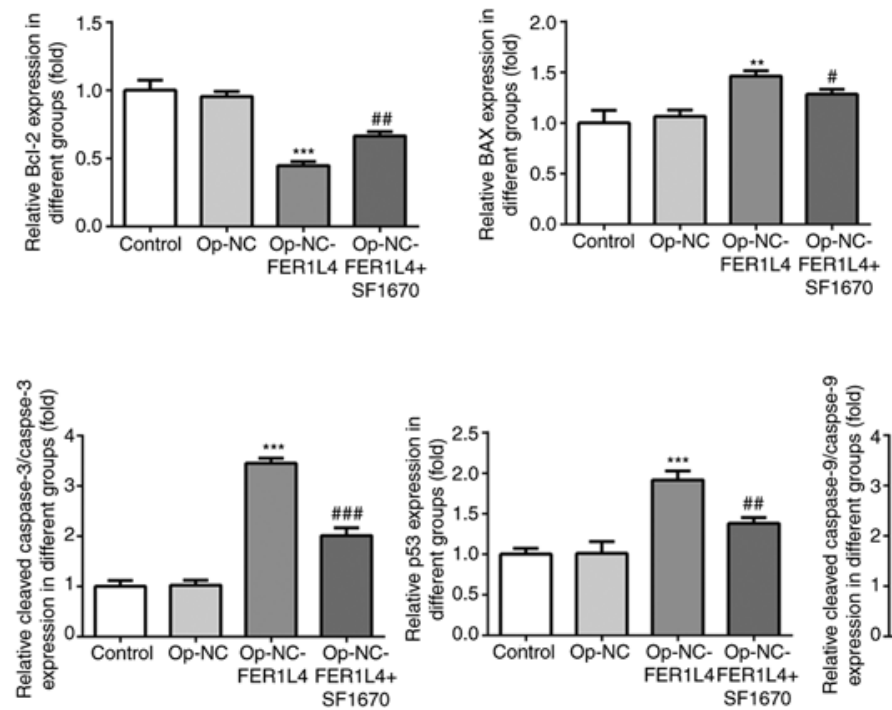

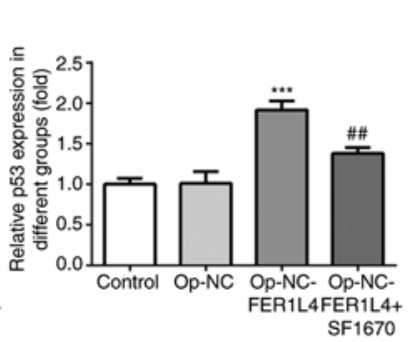

Figure 6. Overexpression of lncRNA FER1L4 promotes apoptosis of lung cancer cells by activating PTEN. The expression of apoptosis-related proteins Bcl-2, BAX, p53, cleaved caspase- 3 and cleaved caspase- 9 were detected by western blotting and densitometric analysis of these proteins is presented. ${ }^{* *} \mathrm{P}<0.01$, ${ }^{* * *} \mathrm{P}<0.001$ vs. Op-NC; ${ }^{\#} \mathrm{P}<0.05,{ }^{\# \#} \mathrm{P}<0.01,{ }^{\# \#} \mathrm{P}<0.001$ vs. Op-NC-FER1L4. FER1L4, Fer-1-like protein 4; PTEN, phosphatase and tension homolog deleted on chromosome ten.

that FER1L4 inhibited cell proliferation and metastasis by inhibiting the PI3K/AKT signaling pathway in lung cancer, which was consistent with our experimental results. Therefore, we can preliminarily conclude that overexpression of lncRNA FER1L4 may promote the apoptosis of lung cancer cells by the PTEN/AKT/p53 pathway. 
In summary it was revealed that the expression of lncRNA FER1L4 was significantly reduced in the plasma of patients with NSCLC and lung cancer cells, and FER1L4 inhibited the proliferation of lung cancer cells and promoted the apoptosis of lung cancer cells through the PTEN/AKT/p53 signaling pathway.

\section{Acknowledgements}

Not applicable.

\section{Funding}

No funding was received.

\section{Availability of data and materials}

The analyzed data sets generated during the present study are available from the corresponding author on reasonable request.

\section{Authors' contributions}

YS designed the study. YS and LO drafted the article. LO was responsible for revising the article. MY and XW analyzed the data. JF, XL and YZ consulted literature and helped conduct the experiments. All authors read and approved the manuscript and agree to be accountable for all aspects of the work in ensuring that questions related to the accuracy or integrity of any part of the work are appropriately investigated and resolved.

\section{Ethics approval and consent to participate}

The study protocol was approved by the Ethics Committee of Northern Jiangsu People's Hospital, and all patients provided written informed consent. All of the procedures were in compliance with The Declaration of Helsinki and relevant policies in China.

\section{Patients consent for publication}

Not applicable.

\section{Competing interests}

The authors declare that they have no competing interests.

\section{References}

1. Mao Y, Yang D, He J and Krasna MJ: Epidemiology of lung cancer. Surg Oncol Clin N Am 25: 439-445, 2016.

2. Zheng RS, Sun KX, Zhang SW, Zeng HM, Zou XN, Chen R, Gu XY, Wei WW and He J: Report of cancer epidemiology in China, 2015. Zhonghua Zhong Liu Za Zhi 41: 19-28, 2019 (In Chinese)

3. Bray F, Ferlay J, Soerjomataram I, Siegel RL, Torre LA and Jemal A: Global cancer statistics 2018: GLOBOCAN estimates of incidence and mortality worldwide for 36 cancers in 185 countries. CA Cancer J Clin 68: 394-424, 2018.

4. Hirsch FR, Scagliotti GV, Mulshine JL, Kwon R, Curran WJ Jr Wu YL and Ares LP: Lung cancer: Current therapies and new targeted treatments. Lancet 389: 299-311, 2017.
5. Bhan A, Soleimani M and Mandal SS: Long noncoding RNA and cancer: A new paradigm. Cancer Res 77: 3965-3981, 2017.

6. Xia T, Chen S, Jiang Z, Shao Y, Jiang X, Li P, Xiao B and Guo J: Long noncoding RNA FER1L4 suppresses cancer cell growth by acting as a competing endogenous RNA and regulating PTEN expression. Sci Rep 5: 13445, 2015.

7. Meng Q, Ren M, Li Y and Song X: LncRNA-RMRP acts as an oncogene in lung cancer. PLoS One 11: e0164845, 2016.

8. Guo F, Cao Z, Guo H and Li S: The action mechanism of IncRNA-HOTAIR on the drug resistance of non-small cell lung cancer by regulating wnt signaling pathway. Exp Ther Med 15: 4885-4889, 2018.

9. Wang F, Chen JG, Wang LL, Yan ZZ, Chen SP and Wang XG: Up-Regulation of LINC00346 inhibits proliferation of non-small cell lung cancer cells through mediating JAK-STAT3 signaling pathway. Eur Rev Med Pharmacol Sci 21: 5135-5142, 2017.

10. Zhang E, Li W, Yin D, De W, Zhu L, Sun S and Han L: C-MycRegulated long non-coding RNA H19 indicates a poor prognosis and affects cell proliferation in non-small-cell lung cancer. Tumour Biol 37: 4007-4015, 2016.

11. Wu J, Huang J, Wang W, Xu J, Yin M, Cheng $\mathrm{N}$ and Yin J: Long non-coding RNA fer-1-like protein 4 acts as a tumor suppressor via miR-106a-5p and predicts good prognosis in hepatocellular carcinoma. Cancer Biomark 20: 55-65, 2017.

12. Wang X, Dong K, Jin Q, Ma Y, Yin S and Wang S: Upregulation of lncRNA FER1L4 suppresses the proliferation and migration of the hepatocellular carcinoma via regulating PI3K/AKT signal pathway. J Cell Biochem 120: 6781-6788, 2019.

13. Yue B, Sun B, Liu C, Zhao S, Zhang D, Yu F and Yan D: Long non-coding RNA fer-1-like protein 4 suppresses oncogenesis and exhibits prognostic value by associating with miR-106a-5p in colon cancer. Cancer Sci 106: 1323-1332, 2015.

14. Liu Z, Shao Y, Tan L, Shi H, Chen S and Guo J: Clinical significance of the low expression of FER1L4 in gastric cancer patients. Tumour Biol 35: 9613-9617, 2014.

15. Fei D, Zhang X, Liu J, Tan L, Xing J, Zhao D and Zhang Y: Long noncoding RNA FER1L4 suppresses tumorigenesis by regulating the expression of PTEN targeting miR-18a-5p in osteosarcoma. Cell Physiol Biochem 51: 1364-1375, 2018.

16. Livak KJ and Schmittgen TD: Analysis of relative gene expression data using real-time quantitative PCR and the 2(-Delta Delta C(T)) method. Methods 25: 402-408, 2001.

17. Wu J, Li L, Wang Y, Ren $X$, Lin $K$ and $\mathrm{He} Y$ : The HSP90/AKT pathway may mediate artemether-induced apoptosis of Cal27 cells. FEBS Open Bio 9: 1726-1733, 2019.

18. Zhang LX, Ding F, Wang CQ, Bing Q, Zhao Z, Wang J and Zhang L: MiR-181a affects myocardial ischemia-reperfusion injury in rats via regulating akt signaling pathway. Eur Rev Med Pharmacol Sci 23: 6292-6298, 2019.

19. Travis-WD, Brambilla E, Nicholson AG, Yatabe Y, Austin JHM, Beasley MB, Chirieac LR, Dacic S, Duhig E, Flieder DB, et al: The 2015 world health organization classification of lung tumors: Impact of genetic, clinical and radiologic advances since the 2004 classification. J Thorac Oncol 10: 1243-1260, 2015.

20. Li Y, Wang Z, Shi H, Li H, Li L, Fang R, Cai X, Liu B, Zhang X and Ye L: HBXIP and LSD1 scaffolded by lncrna hotair mediate transcriptional activation by c-myc. Cancer Res 76: 293-304, 2016.

21. Qi F, Liu X, Wu H, Yu X, Wei C, Huang X, Ji G, Nie F and Wang K: Long noncoding AGAP2-AS1 is activated by SP1 and promotes cell proliferation and invasion in gastric cancer. J Hematol Oncol 10: 48, 2017.

22. Iyer MK, Niknafs YS, Malik R, Singhal U, Sahu A, Hosono Y, Barrette TR, Prensner JR, Evans JR, Zhao S, et al: The landscape of long noncoding RNAs in the human transcriptome. Nat Genet 47: 199-208, 2015.

23. Pan C, Yao G, Liu B, Ma T, Xia Y, Wei K, Wang J, Xu J, Chen L and Chen Y: Long noncoding RNA FAL1 promotes cell proliferation, invasion and epithelial-mesenchymal transition through the PTEN/AKT signaling axis in non-small cell lung cancer. Cell Physiol Biochem 43: 339-352, 2017.

24. Lu Q, Shan S, Li Y, Zhu D, Jin W and Ren T: Long noncoding RNA SNHG1 promotes non-small cell lung cancer progression by up-regulating MTDH via sponging miR-145-5p. FASEB J 32: 3957-3967, 2018.

25. Xia L, Nie D, Wang G, Sun C and Chen G:FER1L4/miR-372/E2F1 works as a ceRNA system to regulate the proliferation and cell cycle of glioma cells. J Cell Mol Med 23: 3224-3233, 2019.

26. Qiao Q and Li H: LncRNA FER1L4 suppresses cancer cell proliferation and cycle by regulating PTEN expression in endometrial carcinoma. Biochem Biophys Res Commun 478: 507-512, 2016. 
27. Ma L, Zhang L, Guo A, Liu LC, Yu F, Diao N, Xu C and Wang D: Overexpression of FER1L4 promotes the apoptosis and suppresses epithelial-mesenchymal transition and stemness markers via activating PI3K/AKT signaling pathway in osteosarcoma cells. Pathol Res Pract 215: 152412, 2019.

28. Sun X, Zheng G, Li C and Liu C: Long noncoding RNA ferllike family member 4 suppresses hepatocellular carcinoma cell proliferation by regulating PTEN in vitro and in vivo. Mol Med Rep 19: 685-692, 2019.

29. Wang Z, Li C, Li Y, Guo X, Yan Z, Gao F and Li C: DpdtbAInduced growth inhibition in human esophageal cancer cells involved inactivation of the p53/EGFR/AKT pathway. Oxid Med Cell Longev 2019: 5414670, 2019.
30. Han Z, Chen F, Ge X, Tan J, Lei P and Zhang J: MiR-21 alleviated apoptosis of cortical neurons through promoting PTEN-Akt signaling pathway in vitro after experimental traumatic brain injury. Brain Res 1582: 12-20, 2014.

31. Chen J: The cell-cycle arrest and apoptotic functions of $\mathrm{p} 53$ in tumor initiation and progression. Cold Spring Harb Perspect Med 6: a026104, 2016.

32. Gao X, Wang N, Wu S, Cui H, An X and Yang Y: Long noncoding RNA FER1L4 inhibits cell proliferation and metastasis through regulation of the PI3K/AKT signaling pathway in lung cancer cells. Mol Med Rep 20: 182-190, 2019. 